\title{
SIMULATION OF LANGMUIR-BLODGETT FILM SURFACE STM IMAGES
}

\author{
V.E. Agabekov, G.K. Zhavnerko \\ Institute of Physical Organic Chemistry, Belarus Academy of Sciences \\ Minsk 220072, Belarus \\ G. BAR AND H.-J. CANTOW
}

Freiburger Materialforschungszentrum, Albert-Ludwigs-Universität Freiburg 79104, Germany

The simulation of the STM image of a hydrocarbon tail of a fatty acid was carried out and compared to the experimental results. The simulation procedure includes calculations of the distribution of an isolated molecule electronic density by the extended Huckel-Hoffmann method. An agreement between the calculated and experimental STM images of closely packed Langmuir-Blodgett film of cobalt behenate on the graphite surface was observed. The weak interactions between the graphite surface and the adsorbed molecules constituting bi- and multilayer Langmuir-Blodgett films can be neglected in simulations of STM images.

PACS numbers: $68.55 .-\mathrm{a}, 71.20 . \mathrm{Rv}, 02.60 . \mathrm{Cb}$

\section{Introduction}

Scanning probe microscopy is an attractive tool for three-dimensional imaging of biological objects, including such model prototypes of biological membranes as Langmuir-Blodgett (LB) films. Interpretation of the scanning tunneling microscopy (STM) images of molecular layers cannot be done efficiently without rational theoretical treatment.

There exist a number of approaches to the understanding of STM images of adsorbate molecules on a substrate [1-3]. In one of the first theoretical attacks for the case of organic adsorbate, Lippel et al. [2] showed a similarity between the frontier molecular orbital electron density distribution and the STM image of copper-phthalocyanine molecules. A more rigorous approach for calculation of the tunneling current between the tip and an adsorbate/substrate system was proposed later by Ou-Yang et al. [3]. We use this approach to interpretation of the STM images of closely packed cobalt behenate LB films on the high oriented pyrolytic graphite (HOPG) surface.

The objective of the present work is to compare the experimentally obtained STM images of organic adsorbates with those predicted by theory. 


\section{Experimental details}

Atomic force microscopy (AFM) images were obtained using Digital Instruments Nanoscope IIIa. The tunneling microscope was the original instrument constructed at Moscow State University [4].

Two types of mono- and multilayer films of cobalt behenate were prepared by: (i) usual LB "vertical" method; (ii) modified "horizontal lift" method, when the monolayer spread on the subphase is deposited on an HOPG when the level of the subphase surface with LB film decreasing below horizontally kept graphite substrate. The monolayers were prepared by spreading chloroform solutions of behenic acid $\left(\mathrm{C}_{21} \mathrm{H}_{43} \mathrm{COOH}\right)$ with a density of $0.5-1 \mathrm{mg} / \mathrm{ml}$ on the subphase of $\mathrm{CoBr}_{2}\left(5 \times 10^{-4} \mathrm{M}\right)$. The layers were compressed to a surface pressure of 20,30 , or $40 \mathrm{mN} / \mathrm{m}$.

\section{Calculation procedure}

Considering that the molecular orbitals are written as linear combinations of atomic orbitals $\chi_{i}$

$$
\Psi_{\alpha}=\sum c_{i}^{\alpha} \chi_{i}
$$

one obtains, in accordance with [3], the following expression for adsorbate contributions to the tunneling current for a given tip-substrate distance:

$$
I \propto \sum_{i, j}^{N} \sum_{m} c_{i} c_{j}\left\langle\chi_{i}|\boldsymbol{H}| d_{m}^{\mathrm{t}}\right\rangle\left\langle d_{m}{ }^{\mathrm{t}}|\boldsymbol{H}| \chi_{i}\right\rangle,
$$

where $d_{m}^{\mathrm{t}}$ denotes the $m$-th $d$-orbital of the tip atom closest to the sample. The molecular orbital coefficients $c_{i}$ and energy levels of the film-forming molecules were calculated by the extended Huckel method [5]. Necessary parameters were taken from Refs. [5-7].

\section{Results and discussion}

A typical image of Co-behenate 1-Y layer is presented in Fig. 1a. The holes in the LB film were used for determination of the layer thickness. The value of $78 \AA$ for the $1-Y$ layer film was obtained, suggesting that the $\mathrm{CoBr}_{2}$ subphase was incorporated in the bilayer film (the thickness of a bilayer film of fully stretched behenic acid molecules is equal approximately to $56 \AA[8]$ ). It was also found that bi- and multilayers exhibit better homogeneity and ordering than monolayers. Unlike the "vertical" deposition method, deposition at $30 \mathrm{mN} / \mathrm{m}$ by the "modified horizontal lift" technique produced practically defect-free layers.

HOPG surface is clearly seen with molecular resolution in Fig. 1b, c. Molecular resolution of the surface of cobalt behenate is obtained in Fig. 1d. Figure 1d differs from the usual STM image of a bare graphite surface because of the presence of an LB film. The experimental STM images suggest the regular two-dimensional arrangement of the molecules. Since the STM images were taken with relatively large tunnel gaps, corrugations usually attributed to the metal ions are not observed. 

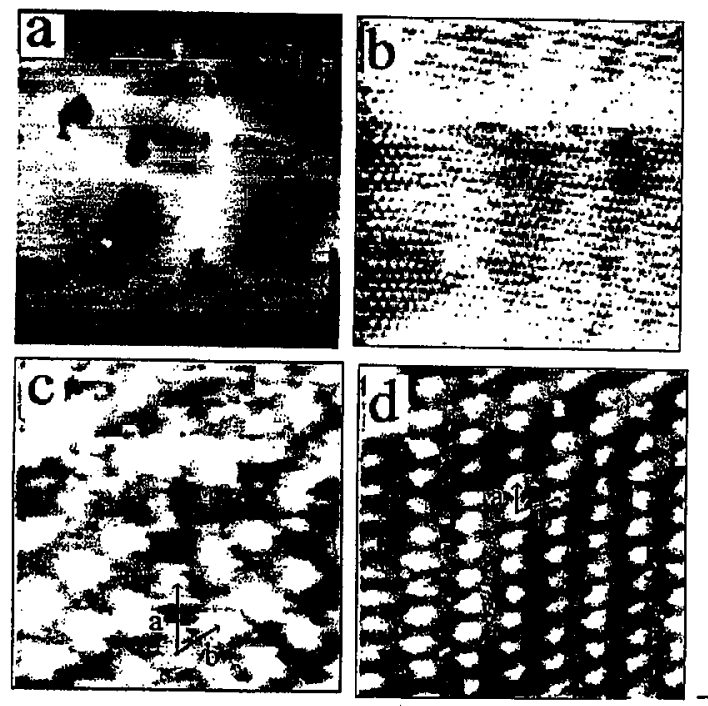

Fig. 1. (a) AFM image $(2 \times 2 \mu \mathrm{m})$ of cobalt behenate LB film (1 bilayer Y-type); (b) and (c) represent the STM images of the HOPG atomic structure observed at scanning areas $130 \times 130$ and $15 \times 15 \AA^{2}$ respectively; (d) STM image $\left(38 \times 38 \AA^{2}\right)$ of cobalt behenate LB film ( 1 bilayer). Distances indicated by arrows are: $a=2.5, b=1.9 \AA$ for (c) case; $a=2.9, b=5.6 \AA$ for (d) case.
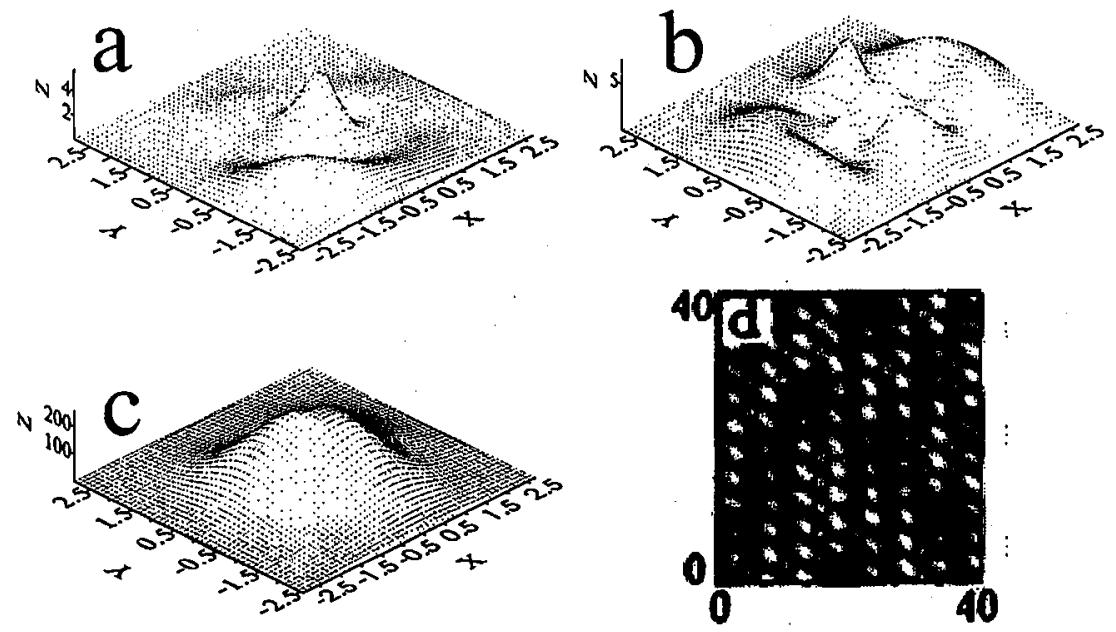

Fig. 2. Calculated contour plots of the constant current STM images of the methyl group of the (a) HOMO, (b) LUMO, (c) sum of all MO's $2 \AA$ above the molecular plane; (d) reconstruction with the (c) case of the STM image of surface close packed methyl groups on an HOPG surface. 
We found a good correlation between the obtained ratio of two-dimensional lattice parameters $b / a=1.93$ (Fig. 1c) and an analogous value $b / a=2$ determined with AFM in monolayers of stearates [9]. The packing density (surface area per molecule) is about $24 \AA^{2}$ which is in a reasonable agreement with the limit surface area of the cobalt behenate film $\left(20.5 \AA^{2}\right)$. It is suggested that the observed corrugations correspond to closely packed alkyl tails of the organic layer. The difference between determined surface area per molecule and limiting one is connected with the slight tilt of alkyl tail relatively to the surface normal.

For the terminal methyl groups of the alkyl tail we calculated three tunneling current distributions using the assumptions that the only orbitals involved in the tunneling process are: (i) the highest occupied molecular orbital (HOMO), (ii) the lowest unoccupied molecular orbital (LUMO), (iii) all molecular orbitals.

The optimized molecular geometries were obtained using Alchemy-II package. The resulting 3D images are shown in Fig. 2a-c. The image in Fig. 2c (the case of all MO's) was found to be in the best agreement with experiment. Images of an LB film on graphite surface shown in Fig. 2d were obtained by superimposing the plot of the current density distribution for selected group of molecule calculated using Eq. (3.2) on the plot of the current density for the graphite surface. A good agreement between Figs. 1d and 2d is clearly seen.

\section{Conclusions}

We observed an agreement between the calculated and experimental STM images of closely packed LB films on the graphite surface. The images of the LB films of fatty acid salts obtained by AFM and STM methods were found to have many similar features. The weak interactions between the graphite surface and the adsorbed molecules constituting bi- and multilayer LB films can be neglected in simulations of STM images.

\section{Acknowledgment}

The authors would like to thank V.N. Staroverov for providing the code to perform the molecular orbital calculations.

\section{References}

[1] J. Tersoff, D.R. Hamann, Phys. Rev. B 31, 805 (1985).

[2] P.H. Lippel, R.J. Wilson, M.D. Miller, Ch. Woll, S, Chiang, Phys. Rev. Lett. 62, 171 (1989).

[3] H. Ou-Yang, R.A. Marcus, B. Kallebring, J. Chem. Phys. 100, 7814 (1994).

[4] Yu.N. Moiseev, V.I. Panov, S.V. Savinov, S.I. Vasil'ev, I.V. Yaminsky, Ultramicroscopy 42/44, 1595 (1992).

[5] R. Hoffmann, J. Chem. Phys. 39, 1397 (1963).

[6] R.S. Mulliken, J. Chem. Phys. 45, 4743 (1966).

[7] R. Rein, N. Fukuda, H. Win, G.A. Clarke, J. Chem. Phys. 45, 4743 (1966).

[8] P. Fromherz, U. Oelschlagel, W. Wilke, Thin Solid Films 159, 421 (1989).

[9] R. Viswanathan, J.A. Zasadzinski, D.K. Schwartz, Science 261, 449 (1993). 